\title{
Efecto de tres densidades de siembra y diferentes dosis de fertilización química en el rendimiento de papa variedad Luyanita INIA-322 propagadas mediante brotes
}

\section{Effect of three seed densities and different doses of chemical fertilization on the yield of potato variety Luyanita INIA-322 propagated by shoots}

\author{
Luis Diovan Valverde Samekash ${ }^{1}$ y Leidy Gheraldinne Bobadilla Rivera ${ }^{1 *}$
}

\section{RESUMEN}

La presente investigación se estableció en la localidad de Levanto en el anexo de Quipachacha a 2356 m.s.n.m donde se evaluaron tres densidades de siembra y diferentes dosis de fertilización química, con el objetivo determinar el efecto de estas variables en el rendimiento de papa variedad Luyanita INIA-322. El diseño experimental utilizado fue diseño factorial en DBCA con nueve tratamientos y tres bloques; siendo los tratamientos compuestos por la combinación de tres densidades de siembra (distancias entre surco y planta de $1 \mathrm{~m}$ x 0,4 m;1 m x 0,3 m y $1 \mathrm{~m}$ x 0,2 m) y tres dosis de fertilización química (N: P: K en dosis 140-120-100; 160-140-120 y 180-160-120). Se realizaron evaluaciones de altura de planta y número de tallos por planta a los 33, 40 y 47 días después de la siembra, para finalmente realizar una evaluación de rendimiento a la cosecha, donde se consideraron variables como producción en kilogramos, número total de tubérculos por planta y número de tubérculos según categoría. Finalmente, se realizó el análisis de varianza y la prueba de Tukey para la comparación múltiples de medias, obteniéndose que el tratamiento $\mathrm{T} 7$ con $24,95 \mathrm{tn} / \mathrm{ha}$ obtuvo el mejor rendimiento de tubérculos por hectárea contrastando con el T3 que tuvo el menor rendimiento con $13,2 \mathrm{tn} / \mathrm{ha}$.

Palabras clave: rendimiento, producción, método de propagación.

\section{ABSTRACT}

The present investigation was established in the town of Levanto in the annex of Quipachacha at 2356 msnm where three seed densities and different doses of chemical fertilization were evaluated, with the objective of determining the effect of these variables on the yield of potato Luyanita variety INIA- 322. The experimental design used was DBCA design with nine treatments and three blocks; The treatments were composed of three planting densities (furrow and plant distances of $1 \mathrm{mx} 0.4 \mathrm{~m}, 1 \mathrm{mx} 0.3 \mathrm{~m}$ and $1 \mathrm{mx} 0.2 \mathrm{~m}$ ) and three doses of chemical fertilization (N: P: $\mathrm{K}$ in doses 140-120-100, 160-140-120 and 180-160-120). Plant height assessments and stem numbers per plant were performed at 33, 40 and 47 days after sowing, to finally perform a crop yield assessment, where variables such as production in kilograms, total number of tubers per plant and number of tubers by category. We performed the analysis of variance and the Tukey test for the comparison of multiple means, obtaining that the T7 treatment with $24.95 \mathrm{tn} /$ ha obtained the best yield of tubers per hectare in contrast with the T3 that had the lowest yield with 13, 2 $\mathrm{tn} / \mathrm{ha}$.

Key words: performance, production, method of propagation.

\footnotetext{
${ }^{1}$ Universidad Nacional Toribio Rodríguez de Mendoza de Amazonas (UNTRM-A), Instituto de Investigación para el Desarrollo Sustentable de Ceja de Selva, Calle Higos Urco N 342-350-356, Calle Universitaria N³04, Chachapoyas, Perú

"Autor de correspondencia. E-mail: lbobadilla@indes-ces.edu.pe
} 


\section{INTRODUCCIÓN}

La papa (Solanum tuberosum L.), es uno de los cultivos alimenticios más importantes tanto en países desarrollados como en vías de desarrollo. La importancia económica de la papa se fundamenta, por su elevada capacidad de producción de sustancias alimenticias por unidad de superficie; es tres veces mayor que en los cereales, lo que permite suplir de alimento a un elevado número de familias (Coleman et al., 2001). Es así que, en el Perú, la papa es cultivada por aproximadamente por 710000 familias, según el IV censo nacional agropecuario, además, es producida en 19 regiones del país (DGPA, 2017). Así mismo, de los cultivos transitorios, la papa ocupó el primer lugar en superficie cultivada con 367,7 mil hectáreas (INEI, 2013).

La región amazonas obtuvo para el año 2016, rendimientos promedio de $15 \mathrm{tn} / \mathrm{ha}$, igualando el promedio nacional (MINAGRI, 2017). Sin embargo, en la provincia de Chachapoyas es conocida la existencia de zonas donde la producción es menor al promedio nacional.

En la productividad del cultivo intervienen una serie de factores como que siendo manejados correctamente van a conducir al éxito en la producción de papa. El uso de semilla de buena calidad es importante, ya que, se emplea la propagación vegetativa (por medio de sus tubérculos). Una semilla que no esté en condiciones sanitarias, físicas y fisiológicas adecuadas, producirá germinación desuniforme, un pobre desarrollo de plantas y bajos rendimientos y se corre el riesgo de diseminar, involuntariamente, plagas y enfermedades (Montesdeoca, 2005). La obtención de esquejes de brotes, al igual que otras técnica biotecnológicas, se presenta como alternativa para la disminución del riesgo de propagar patógenos en la semilla (Cotes y Ñustez, 2001), esta técnica consiste en, inducir la germinación de los brotes en los tubérculos para luego separarlos de la estructura de la semilla y enraizarlos en material estéril (INIAP, 1993). Los niveles de fertilización adecuados es otro factor importante a tener en cuenta, el uso de los fertilizantes se hace necesario con el fin de hacer rentable la actividad agrícola, debiendo incluir la dosis apropiadas de N, P y K, los cuales al mismo tiempo de nutrir a la planta, otorgan también características de resiliencia frente al ataque de plagas y enfermedades (Ros et al., 2008); así mismo la densidad de siembra influye en el rendimiento final de la plantación, se estima que se deben tener entre 30000 a 35000 plantas por hectárea. Es decir 3 a 3,5 plantas por metro cuadrado, con surcos distanciados entre 0,80 a 1,00 m(Tapia y Fries, 2007).

En la actualidad existe mayor demanda de alimentos en todo el mundo, por lo que la única solución viable es el incremento de los rendimientos por unidad de área y esto se obtiene a través de la generación de tecnología (uso de nuevas variedades, nuevas formas de propagación, entre otros), que estén acorde a los cambios y necesidades reales (Coleman et al., 2001). En ese sentido la variedad Luyanita INIA 332, variedad lanzada en el 2014, cuenta con alto rendimiento y resistencia a rancha de la papa (INIA, 2014).

En ese orden de ideas se planteó evaluar el efecto de la densidad de siembra y la aplicación diferentes dosis de fertilización en plantas de papa de la variedad INIA 332-Luyanita propagadas a partir de brotes.

\section{MATERIALES Y METODOS}

El ensayo se realizó en la localidad de Quipachacha, distrito de Levanto, provincia de Chachapoyas esta localidad está ubicada a una altitud de entre 2400 y 2600 msnm. El ensayo constó de 9 tratamientos manejados en un diseño en bloques completos al azar, estos tratamientos estuvieron definidos por tres dosis de fertilización (N: P: K en dosis 140-120-100; 160-140-120 y 180160-120) y tres densidades de siembra (distancias entre surco y planta de $1 \mathrm{~m}$ x 0,4 m; $1 \mathrm{~m}$ x 0,3 m y $1 \mathrm{~m} \mathrm{x}$ $0,2 \mathrm{~m})$. El terreno de cultivo fue acondicionado mediante desmalezado y arado, para el posterior trazo del mismo según el diseño planteado.

Para efectos del experimento se utilizaron brotes de papa de la variedad INIA 332-Luyanita, que fueron extraídos de tubérculos previamente germinados cuando tuvieron una altura de aproximadamente $2 \mathrm{~cm}$, 
desinfectados con enziprom, curzaten (cymoxanil + mancozeb), cipermex (Cipermetrina); estos brotes fueron tratados en una solución de inductores de enraizamiento y desinfectantes. Para su selección, los brotes fueron trasplantados en tubetes de $115 \mathrm{~cm}^{3}$ con sustrato que contenía cuatro partes de tierra agrícola, dos partes de compost, dos partes de arena de río y una parte de cascarilla de arroz.

Una vez los brotes alcanzaron la altura de $5 \mathrm{~cm}$. aproximadamente se procedió a instalarlos en campo definitivo según los distanciamientos establecidos por los tratamientos. La fertilización también se efectuó según lo definido en los tratamientos utilizando Urea como fuente de nitrógeno $(\mathrm{N})$, fosfato diamónico como fuente de fósforo $(\mathrm{P})$ y cloruro de potasio con fuente potasio $(\mathrm{K})$. La fertilización se realizó fraccionando el N y K 50\% a la siembra y $50 \%$ al deshierbo, el fosfato diamónico se aplicó el 100\% al momento de la siembra.

El área total del terreno fue de $636 \mathrm{~m}^{2}$, se consideró 3 bloques de, los cuales estaban conformados por 9 parcelas con una distancia de separación entre bloques 1,5 $\mathrm{m}$ y parcelas de $1,0 \mathrm{~m}$, cada unidad experimental tuvo un área de $15 \mathrm{~m}^{2}$ ( $3 \mathrm{~m}$ x $\left.5 \mathrm{~m}\right)$. Cada unidad experimental fue una parcela de 4 surcos, donde se evaluó 18,18 y 25 plantas para las densidades 1,2 y3 respectivamente según la muestra estimada. Los tratamientos se detallan en la tabla 1.

Tabla 1. Tratamientos instalados en la investigación

\begin{tabular}{cccc}
\hline Tratamientos & $\begin{array}{c}\text { Distancia } \\
\text { entre } \\
\text { planta } \\
\text { (m) }\end{array}$ & $\begin{array}{c}\text { Distanci } \\
\text { a entre } \\
\text { surco } \\
\text { (m) }\end{array}$ & $\begin{array}{c}\text { Dosis de } \\
\text { fertilización } \\
\text { N-P-K }\end{array}$ \\
\hline T1 & 0,4 & 1 & $140-120-100$ \\
T2 & 0,4 & 1 & $160-140-120$ \\
T3 & 0,4 & 1 & $180-160-120$ \\
T4 & 0,3 & 1 & $140-120-100$ \\
T5 & 0,3 & 1 & $160-140-120$ \\
T6 & 0,3 & 1 & $180-160-120$ \\
T7 & 0,2 & 1 & $140-120-100$ \\
T8 & 0,2 & 1 & $160-140-120$ \\
T9 & 0,2 & 1 & $180-160-120$ \\
\hline
\end{tabular}

Las evaluaciones se realizaron en 5 plantas escogidas al azar para cada tratamiento, esto se realizó cada 7 días y en tres oportunidades. Para esta sección de lineamiento se tuvo en cuenta parámetros como evaluación de altura de planta, número de tallos por planta y vigor de la planta.

Para la evaluación del rendimiento se tomaron en cuenta las densidades de siembra. De los 4 surcos que tuvo la parcela se cosecharon solo las plantas de los dos surcos centrales. La cosecha, consistió en extraer, contar y colocar los tubérculos en bolsas para ser pesados y finalmente fueron seleccionados por categorías.

Tabla 2. Clasificación de semilla según peso de tubérculo

\begin{tabular}{cc}
\hline Clase & Peso \\
\hline Gruesa & Tubérculos mayores de 80 gramos \\
Primera & Tubérculos entre 60 a 80 gramos \\
Segunda & Tubérculos entre 40 y 59 gramos \\
Tercera & Tubérculos entre 20 a 39 gramos \\
Descarte & Tubérculos menores de 20 gramos \\
& \multicolumn{2}{c}{ y enfermos } \\
\hline
\end{tabular}

Fuente: Ministerio de agricultura, 2011

Luego de obtenidos los datos fueron procesados y se realizó el análisis de varianza y una vez encontradas diferencias significativas se procedió a realizar una prueba de comparación múltiple de Tukey.

\section{RESULTADOS}

De las evaluaciones realizadas en el ensayo, se obtuvieron datos que luego de ser tabulados, y procesados arrojaron los resultados que a continuación se presen$\tan$.

\section{Altura de planta alcanzada}

Como se observa en la figura 1, en referencia a la altura de planta los datos demuestran que, fue el tratamiento 9 (T9) el que obtuvo los mejores promedios a los 33 días, 40 días y 47 días con 45,6 cm, 53,2 cm y 63,93 cm respectivamente, los días de evaluación calculan desde el día de la siembra; así mismo los tratamientos con menores promedios de altura fueron el T3 a los 33 días después de la siembra con 37,53 cm y el T2 a los 40 y 47 días después de la siembra con 45,73 cm., y $54,2 \mathrm{~cm}$. respectivamente. Se evidencia además, que los intervalos no tienen una amplitud marcada, es así que en la observación a los 47 días, los demás trata- 
mientos alcanzaron alturas intermedias con $54,47 \mathrm{~cm}$; $55,60 \mathrm{~cm} ; 55,80 \mathrm{~cm} ; 56,07 \mathrm{~cm} ; 56,67 \mathrm{~cm}$ у $58,87 \mathrm{~cm}$ para los tratamientos T1, T5, T3, T6, T4 y T8 respectivamente.

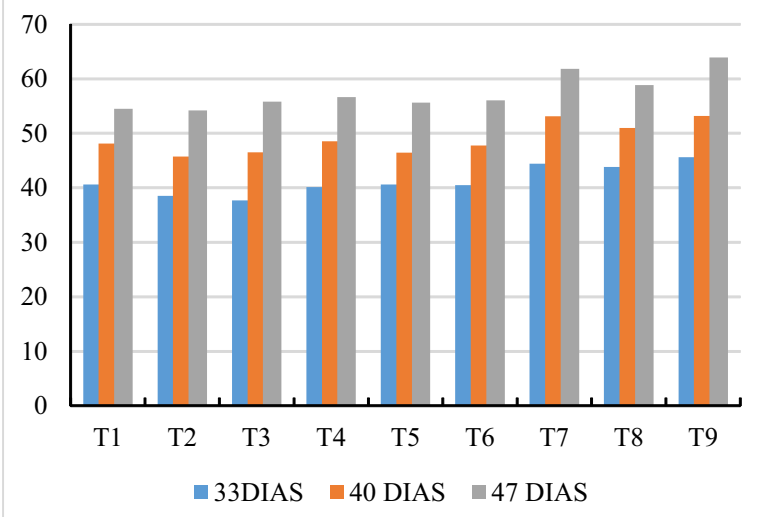

Figura 1. Altura de planta alcanzada a los 33, 40 y 47 días después de la siembra, se detallan las alturas de planta en promedio alcanzadas por cada tratamiento.

\section{Número de tallos por planta}

Por otro lado en la figura 2 podemos encontrar que, en la observación a los 47 días después de la siembra, aun no teniendo una altura de planta superior, el tratamiento 6 (T6) es el que presenta un mayor número de tallos por planta con 5,40 tallos en promedio, le sigue el T9 con 5,33 tallos por planta en promedio, y en contraste el tratamiento con menor número de tallos por planta fue el T7 con 4,13 tallos por planta.

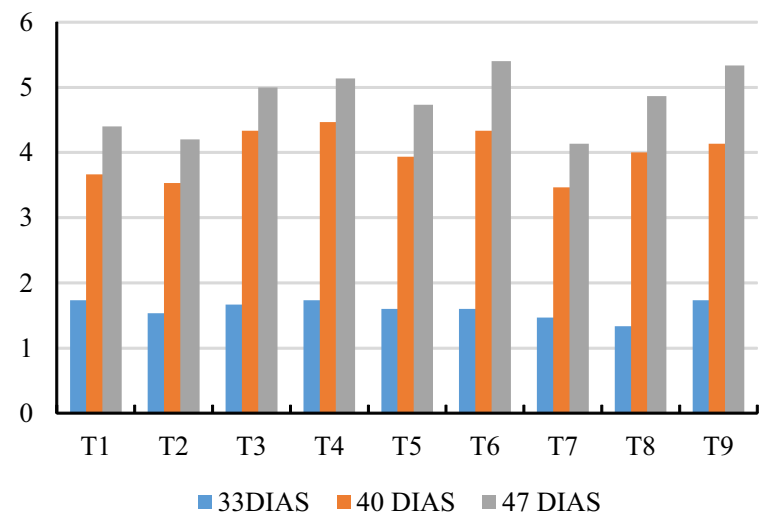

Figura 2. Número de tallo por planta a los 33, 40 y 47dias después de la siembra.

\section{Producción de tubérculo por planta en kilogramos}

En la figura 3 se muestra el resultado de la evaluación de producción, encontrándose que, respecto a la producción promedio de tubérculo alcanzados por planta, el mayor rendimiento de tubérculo fue obtenido por el tratamiento (T2) con $0,59 \mathrm{Kg}$ y el menor rendimiento de tubérculo obtenido fue el tratamiento (T8) con $0,39 \mathrm{Kg}$., los demás tratamientos obtuvieron rendimiento de tubérculos intermedios.

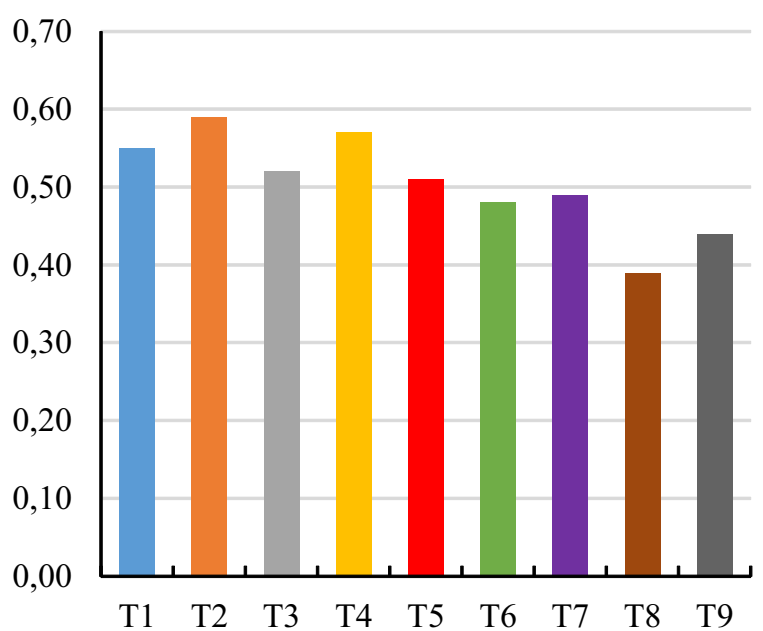

Figura 3. Producción de tubérculo de papa por planta en promedio expresado en $\mathrm{kg}$.

\section{Rendimiento de tubérculo (tn/ha)}

En la figura 4 se muestra los valores alcanzados en rendimiento, expresado en th/ha, en donde se observa que el tratamiento (T7) logró obtener 24,95 tn/ha siendo el máximo rendimiento alcanzado, mientras que, el menor rendimiento fue obtenido por el tratamiento (T3) con 13,2 tn/ha, los demás tratamientos obtuvieron rendimientos contenidos en ese rango con 13,2 tn/ha; 14,88 tn/ha; 16,27 tn/ha; 17,27 tn/ha; 19,17 tn/ha y 19,8 tn/ha para los tratamientos T3, T2, T6, T5, $\mathrm{T} 4, \mathrm{~T} 8$ respectivamente.

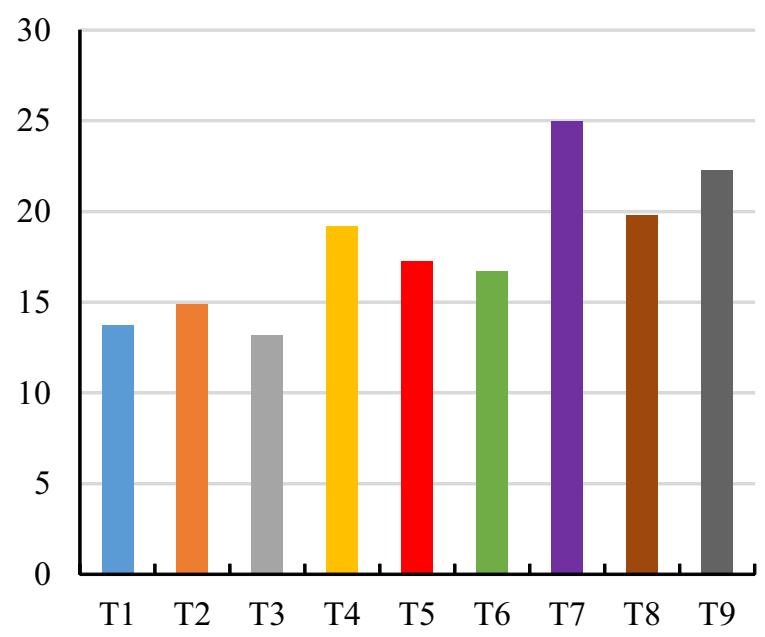

Figura 4. Rendimiento de tubérculo a nivel experimental expresado en $\mathrm{tn} / \mathrm{ha}$. 
Número total de tubérculos por tratamiento (Tu-

\section{bérculo/planta)}

Por su parte la figura 5 muestra los datos obtenidos del conteo de tubérculos alcanzados por planta a la cosecha, se puede observar que el mayor número de tubérculos fue alcanzado por el tratamiento (T4) con 13,87 en segundo lugar se encuentra el tratamiento (T3) con 11,60 y el tratamiento con el menor número de tubérculo fue el tratamiento ocho (T8) con 9,07. Los demás tratamientos se encuentran en un rango contenido en este intervalo.

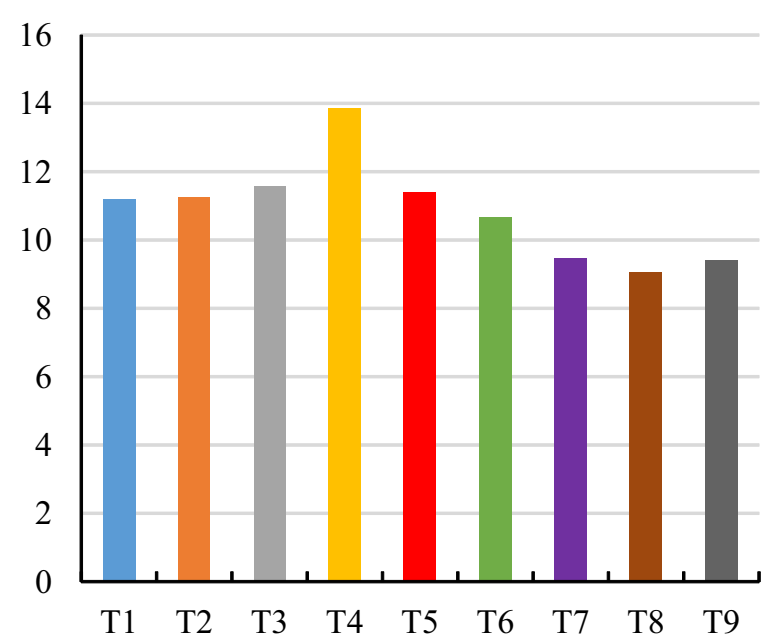

Figura 5. Número total de tubérculos por planta obtenidos en cada tratamiento.

\section{DISCUSION}

En cuanto a la altura de planta, el valor con mayor altura alcanzado lo reportó el tratamiento (T9) con $63,9 \mathrm{~cm}$, seguido por el tratamiento (T7) con $61,8 \mathrm{~cm}$, comparados con los demás tratamientos donde muestran un comportamiento similar; este resultado podría deberse a la competencia por luz ya que se observa que a medida que la densidad de plantas aumenta, aumenta también el enlongamiento de las plantas, por lo que se asume que las plantas con mayor densidad tiene un comportamiento de seguir su crecimiento, debido al fototropismo existente en las mismas, al respecto Rivera (1991) indica que a partir de un determinado momento aumenta el crecimiento de las plantas y desarrollo en las mayores densidades, debido a que existe competencia por luz. Además la misma relación se observa con respecto a la fertilización ya que se observa que el T9 es el que recibió mayores dosis de fertilización y es en consecuencia el tratamiento que obtuvo los mejores promedios de rendimiento.

Para el caso del número de tallos por planta se encontró que el tratamiento (T6) obtuvo 5,40 tallos, alcanzando el mejor promedio, seguido del tratamiento (T9) con 5,33 tallos. En referencia a estos datos Zamora et al. (2008), en su investigación evaluación de cinco fuentes orgánicas sobre el desarrollo vegetativo y rendimiento del cultivo de papa, coincide obteniendo datos similares y mencionó que la fertilización química obtuvo promedios superiores con 4,74 tallos por planta. Por otro lado Cisneros y Herrera (1987) señalan que el mayor número de tallos por tubérculos fue alcanzado por una semilla de 56 a $65 \mathrm{~mm}$, también señalan que una mayor cantidad de brotes coincide con una mayor cantidad de tallos y que el número de tubérculos se incrementan. De acuerdo a estos resultados y referencias se asume que la proporción de número de tallos depende del tamaño y tipo de semillas, lo que se estima que a mayor número de brotes por tubérculo semilla será mayor la cantidad de tallos. Sin embargo, en semillas vegetativas propagadas mediante brotes los tallos contados corresponderían a ramas vegetativas, por lo que dichos tallos no dieron origen a la tuberización.

En cuanto a la variable de rendimiento de tubérculo por planta se encontró que el tratamiento 2 (T2) obtiene $0,59 \mathrm{~kg}$. siendo el mejor rendimiento de tubérculo por planta; le sigue el tratamiento 4 (T4) con $0,57 \mathrm{~kg}$, así mismo los tratamientos (T1), (T3) y (T5) quienes alcanzaron rendimientos de $0,55 \mathrm{~kg}, 0,52 \mathrm{~kg}$ y $0,51 \mathrm{~kg}$ respectivamente. Finalmente el tratamiento ocho (T8) obtiene solo 0,39 kg. Que lo convierte en el rendimiento más bajo. Sin embargo realizando el cálculo para el rendimiento en tn/ha, en la Figura 4 se observa que el tratamiento siete (T7) obtiene 24,9 tn/ha, mostrando ser el mejor rendimiento, en segundo lugar el tratamiento nueve (T9) con 22,25 tn/ha, En ese sentido podemos enunciar que, aun teniendo mejores índices de producción por planta a menores densidades, no se tiene un rendimiento óptimo expresado en tn/ha, sin 
embargo al aumentar la densidad de siembra, so obtienen mejores rendimientos, aun cuando los índices de producción por planta disminuyen.. En ese sentido Domínguez (2011), en su trabajo evaluó las densidades de siembra y niveles de fertilización química, obteniendo a una densidad de 1,1 x 0,4 m y con la fertilización 200-500-180 kg/ha de N-P-K una producción de 33,78 T/Ha; mientras que en último lugar se ubicó la densidad de 1 x 0,25 m con la fertilización 100-250$90 \mathrm{~kg} / \mathrm{ha}$ de N-P-K produciendo 13,20 T/Ha, lo que difiere del presente trabajo ya que se muestra que el efecto de aumentar la densidad no es favorable para el rendimiento final del cultivo, mientras que en el presente ensayo se demuestra lo contrario.

Los resultados obtenidos para la variable número de tubérculo por planta muestra al tratamiento (T4) con 13,85 seguidamente del tratamiento (T3) con 11,62 los cuales obtienen mayor número de tubérculos por planta, a diferencia del tratamiento (T8) que solo obtuvo 9,04 tubérculos por planta siendo el tratamiento con menor número de tubérculos, estos resultados evidencian que la densidad de planta está incidiendo directamente sobre esta variable, mas no lo hace así la fertilización ya que se observa que el T3 obtuvo un menor número de tubérculos teniendo mayor dosis de fertilización, a diferencia del T4 que recibió menor dosis de fertilización y una densidad mayor, obteniendo mejores promedios de tubérculos por planta., Zamora et al. (2008), mencionan que la densidad es un factor determinante para el número total de tubérculo por planta.

\section{V.CONCLUSIONES}

En referencia a la densidad de siembra, se encontró que los mayores rendimientos de tubérculo de papa por hectárea se obtuvieron en el tratamiento T7 $(0,20$ $\mathrm{m}$ entre planta y $1,0 \mathrm{~m}$. entre surco).

El mayor rendimiento en toneladas de papa por hectárea, fue alcanzado por el tratamiento T7 (dosis 140120-100 de N-P-K), esta dosis de fertilización química obtuvo la mejor respuesta usando úrea, fosfato diamónico y cloruro de potasio.

En cuanto al rendimiento de tubérculo por hectárea se tiene que el tratamiento 7 a una densidad de 50000 plantas por hectárea y una dosis de 140-120-100 de N P K alcanzó el mayor rendimiento de toneladas $(24,95$ tn/ha) y el tratamiento 3 (T3) a una densidad de 25000 plantas por hectárea y una dosis de 180-160-140 de N P K alcanzo el menor rendimiento en toneladas por hectárea.

La metodología de propagación por brotes se convierte en una importante herramienta alternativa a la propagación convencional de este tubérculo, en el presente trabajo se obtuvo un $100 \%$ de prendimiento en campo definitivo.

\section{REFERENCIAS BIBLIOGRÁFICAS}

Coleman, W. K., D. J. Donnelly y S.E. Coleman. 2001. "Potato Microtubers as Research Tools: A Review". American Journal of potato Research 78: 47-55.

Cotes, J. M. y C.E. Ñustez. 2001. "Evaluación de dos tipos de esquejes de brote en la producción de semilla prebásica de papa criolla (Solanum phureja Juz et. Buk) variedad yema de huevo". Agronomia colombiana 18(1-2): 713.

DGPA (Derección General de Políticas Agrarias). 2017. Papa: Características de la produción nacional y de la comercialización en Lima Metropolitana. Lima(Perú): MINAGRI.

Domínguez, D. E.. 2011. Evaluación de densidades de siembra y niveles de fertilización química en la producción de semilla en tres genotipos de papa (Solanum tuberosum) Mejia-Pichincha. Quito(Ecuador): INAIA.

INEI (Instituto Nacional de Estadistica e Informática). 2013. Resultados definitivos: IV censo nacional agropecuario 2012. Lima (Perú): Punto Y Grafía.

INIA (Instituto Nacional de Innovación Agraria). 2014. Lanzamiento de nuevas variedades de papa para el Norte del Perú. Lima (Perú): I N I A. R e c u p e r a d o d e : http://agraria.pe/noticias/inialiberara-nueva- 
variedad-de-papa-\%E2\% 80\%9Cinia-322--

luyanita $\%$ E2\%80\%9D-es- 6195

INIAP (Instituto Nacional Autónomo de Investigaciones Agropecuarias). 1993.

"Producción de semilla de papa con alta calidad sanitaria a partir de cultivos de tejidos". Revista Pracipa. 73.

MINAGRI (Ministerio de Agricultura y Riego). 2017. Anuario estadístico de la producción agrícola y pecuaria 2016. Lima (Perú): MINAGRI.

MINAGRI. 2011. Buenas prácticas agrícolas en el cultivo de papa. Chachapoyas (Perú): MIANGRI.

Montesdeoca, F. 2005. Guía para la producción, comercialización y uso de semilla de papa de calidad. Quito (Ecuador). PNTR-INIAPProyecto Fortipapa.

Rivera, R. 1991. "Densidad de plantacion y aprovechamiento de fertilizante nitrogenado en el cultivo del cafeto, variedad caturra, sobre suelos ferraliticos rojo compactados". Cultivos Tropicales 12 (3): 5-8.

Ros, B., V. Mohler, G. Wenzel, y F. Thümmler. 2008. "Phytophthora infestans-triggered response of growthand defense-related genes in potato cultivars with different levels of resistance under the influence of nitrogen availability". Physiologia Plantarum 133: 386-399.

Tapia, M. E. y A.M. Fries. 2007. Guia de campo de los cultivos andinos". Lima (Perú): FAO Y ANPE.

Zamora, F., D. Tua y D. Torres. 2008. "Evaluación de cinco fuentes orgánicas sobre el desarrollo vegetativo y rendimiento del cultivo de papa". Agronomía Tropical 58 (3): 233-243. 